\title{
The effect of response competition on functional hemispheric asymmetries for global/local processing
}

\author{
RONALD HÜBNER and PETER MALINOWSKI \\ Universität Konstanz, Konstanz, Germany
}

\begin{abstract}
It is widely assumed that the cerebral hemispheres differ in their capacity for processing the global and local levels of hierarchical stimuli. However, corresponding visual-field (VF) effects in response time studies did not show up under all circumstances. In the present article the role of response conflict between the levels for the occurrence of these effects is investigated. Three experiments with hierarchical letters are reported, in which the absolute and/or the relative interference between the stimulus levels was varied. It turned out that $\mathrm{VF}$ effects occurred only for conflicting stimuli and only when there was at least a certain amount of absolute interference, whereas variations of the relative interference had no effect in this respect. These results suggest that there is a qualitative relationship between interference and VF effects. A possible explanation is provided by the assumption that the hemispheres are functionally equivalent with respect to early stimulus representations, whereas they differ in their efficiency for integrating letter identity and stimulus level.
\end{abstract}

An intensively investigated question is how the human visual system selects, processes, and integrates the different features of a stimulus. Physiological (e.g., Livingstone \& Hubel, 1988; Zeki \& Shipp, 1988) as well as experimental (e.g., Humphreys \& Boucart, 1997; Treisman, 1988) data indicate that individual features are processed, at least to some extent, in separate modules or pathways in the brain. The outputs of these modules are then directed to response units or to higher processing stages for their integration. In this respect even the cerebral hemispheres have been considered as modules, specialized for the processing of certain features. One of these specializations, which is the topic of the present article, is concerned with the processing of the global and the local level of hierarchical stimuli. Many objects are composed of parts, and it is possible to attend and respond selectively either to their global shape or to the form of their elements. Examples of hierarchical stimuli with letters on each level are shown in Figure 1. With respect to global/local processing, it has been proposed that the left hemisphere (LH) is specialized for processing local forms, whereas the right hemisphere (RH) processes global forms more efficiently.

A widely applied method for testing this hypothesis is to present hierarchical stimuli either in the right visual field (RVF) or in the left visual field (LVF) and to require speeded responses either to the global or to the local stim-

This research was supported by a grant from the Deutsche Forschungsgemeinschaft (DFG) to R.H. as part of a research group (Ro $805 / 11-1)$. We thank Albrecht Inhoff, Richard Ivry, and two anonymous reviewers for their helpful comments. Correspondence should be addressed to R. Hübner, Universität Konstanz, Fachbereich Psychologie, Fach D29, D-78457 Konstanz, Germany. ulus level. When the proposed hemispheric asymmetries exist, the latencies should show an interaction between visual field (VF) and target level. More specifically, the local level should be processed faster for stimuli presented in the RVF than for those presented in the LVF, whereas the opposite should hold for the global level. Indeed, numerous studies found such interactions (e.g., Hübner, 1997, 1998; Robertson, Egly, Lamb, \& Kerth, 1993).

While response time data provide only indirect evidence for functional hemispheric asymmetries, there is more direct support from studies using functional imaging techniques (PET, positron emission tomography; fMRI, functional magnetic resonance imaging) as well as from lesion studies (Fink, Halligan, et al., 1996, 1997; Fink, Marshall, Halligan, \& Dolan, 1999; Martinez et al., 1997; Robertson \& Lamb, 1991).

However, there are also a number of studies in which functional hemispheric differences were absent or even in the opposite direction (e.g., Boles, 1984; Boles \& Karner, 1996; Fink et al., 1999; Fink, Marshall, Halligan, Frith, \& Frackowiak, 1997; Heinze, Hinrichs, Scholz, Burchert, \& Mangun, 1998; Van Kleeck, 1989). Up to now it is largely unknown what factors are responsible for the heterogeneous and inconclusive results, because the studies differ in a number of sensory and attentional conditions (see Fink et al., 1999; Hübner, 1997, 1998; Ivry \& Robertson, 1998; Robertson \& Lamb, 1991). In an attempt to investigate this issue, Hübner (1997) showed that, for instance, randomizing the target levels within an experimental block increased the asymmetries relative to a condition with constant target levels.

Another factor that seems to modulate the VF effects, at least in response time studies, is response conflict (e.g., 
Outline

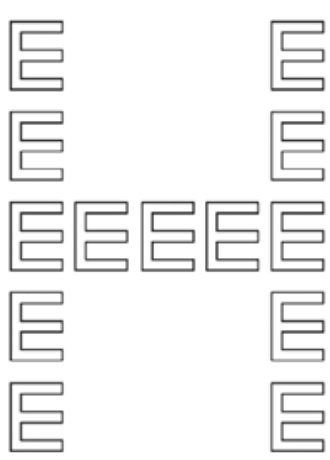

Line

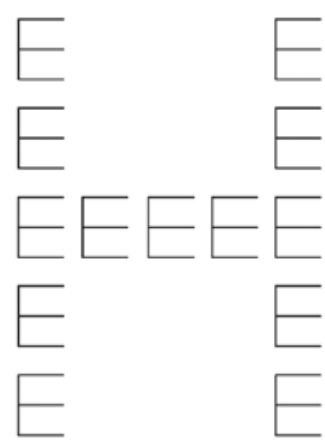

Color

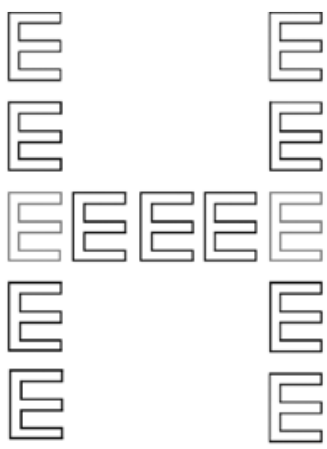

Figure 1. Examples of the different stimulus types used in the experiments. On the screen, the background was black and the lines were white. The gray letters in the color stimuli were presented in yellow.

Hübner, 1998; Kitterle, Christman, \& Conesa, 1993). Usually, conflicts occur for stimuli whose information at each stimulus level is associated with a different response. For instance, if we regard the stimuli in Figure 1, they would be conflicting if the letters " $E$ " and " $H$ " would require different responses. Already Van Kleeck (1989) observed in his meta-analysis that for conflicting stimuli, five out of six studies showed VF effects, whereas only two of the five studies using nonconflicting stimuli showed an effect. At first glance this result seems surprising. How can VF effects with respect to global/local, which is obviously a perceptual dimension, be affected by response conflicts? However, Van Kleeck offered a straightforward explanation. He assumed that both hemispheres are capable of processing the information at both levels, but with different efficiency (see Zaidel \& Rayman, 1994). Increased VF effects for conflicting stimuli are then a direct consequence. Consider, for instance, the case where responses to the local level are required. When a conflicting RVF stimulus is processed in the $\mathrm{LH}$, then an optimally processed local level competes with a suboptimally processed global level, which should hardly affect the response time relative to that for nonconflicting stimuli. On the other hand, the local level of a conflicting LVF stimulus is not only processed suboptimally in the $\mathrm{RH}$, but has also to compete with an optimally processed global level. Consequently, in this case the response time to the local level should be increased considerably relative to a situation with nonconflicting stimuli. Analogous reasoning can be applied to the global level.

This asymmetric pattern of interference implies that VF effects should be larger for conflicting than for nonconflicting stimuli. In other words, response competition between the levels serves as an amplifier for VF effects. Thus, if there are hemispheric asymmetries for global/local processing, the corresponding VF effects are amplified by response competition, which might explain why the effects were observed more frequently for conflicting than for nonconflicting stimuli.
The aim of the present article was to investigate the relation between response conflict, or interference, as we will also call this effect, and VF effects for global/local processing. First, we examine whether the amplifier hypothesis is valid. However, as counterpart, we also consider the alternative hypothesis that response conflicts do not act as an amplifier, but that they are crucial for obtaining VF effects. Thus, according to this hypothesis, interference acts like a catalyst.

The amplifier hypothesis obviously predicts a quantitative relation between VF effects and the amount of interference between the levels. The greater the response conflict, the larger the VF effects. It should be noted, however, that this hypothesis is based on the assumption that VF effects are also be present, even if to a lesser extent, for nonconflicting stimuli.

To test the predictions, a series of experiments were conducted in which the absolute interference and/or the relative interference between the levels was manipulated. For compatibility with our former articles, we will denote nonconflicting and conflicting stimuli as "consistent" and "inconsistent," respectively. Usually, the overall performance is reduced for inconsistent stimuli relative to that for consistent ones. On the other hand, the relative amount of reduction for each level of a hierarchical stimulus depends on its dominance or salience. Under many conditions the global level is more salient, and, therefore, responses to the global level are faster than those to the local level. Given such a global response time advantage, it is likely that there is also a global interference advantagethat is, more global-to-local interference than vice versa.

The strong global dominance in his experiments led Navon (1977) to propose a strict sequential processing order from global to local. Meanwhile, however, it has been shown that the relative salience of the levels depends on stimulus factors such as absolute and relative size, element density, and so on. Certain conditions even produce faster responses to the local level (e.g., Boer \& Keuss, 1982; Miller, 1981). Moreover, under some conditions a global re- 
sponse time advantage occurred together with a local interference advantage (e.g., Hübner, 1997; Lamb \& Robertson, 1988). Altogether, these results indicate that the global and local types of information are processed in parallel, rather than in a fixed order.

On the basis of the hypothesis of hemispheric specialization for global/local processing, we express VF effects for the local level by subtracting the mean response time for the local level of RVF stimuli from that for the local level of LVF stimuli. Analogously, the VF effect for the global level was computed by subtracting the mean latencies for the global level of LVF stimuli from that for the global level of RVF stimuli. Thus, for each level, a positive value indicates that the VF effect points in the expected direction.

\section{EXPERIMENT 1}

In the first experiment we tried to vary the amount of absolute interference between the levels by blocking versus randomizing the target levels. Although a beneficial effect of randomized target levels on VF effects has already been shown in Hübner (1997), its relation with response conflicts remains unclear. Thus, here, we expected not only that the VF effects would increase under randomized target levels, but also that the interference would increase. Such a co-occurrence would support the hypothesis that VF effects are amplified by interference, which is increased under randomized levels. Furthermore, in Hübner (1997) a between-subjects design was used, whereas here, the results should be replicated with a within-subjects design.

Additionally, the relative amount of interference between the levels was varied by manipulating the relative salience of the levels-employing two different types of hierarchical letters as stimuli. One type were global letters constructed from local outline letters, whereas the other type was constructed from local letters composed of simple lines. Examples of the line and outline stimuli are shown in Figure 1. We assumed that the relative salience of the levels differs between the stimuli. However, since we had no prior experience with the line stimuli, we did not know in advance in what direction the difference would be. Therefore, no specific hypothesis was stated. Rather, on the basis of the amplifier hypothesis it was expected that any variation of the relative interference between the stimulus types should produce a corresponding variation of VF effects. For instance, when one stimulus type produces more global-to-local interference than the other type, the former should also produce larger local VF effects and smaller global VF effects than the latter.

\section{Method}

Subjects. Sixteen persons ( 8 female, mean age 24.9 years; 8 male, mean age 25.9 years) participated in the experiment. All were righthanded (by self-report) and had normal or corrected-to-norm al vision.

Apparatus. The stimuli were presented on a 21-in. color monitor (Sony) with a resolution of $1,280 \times 1,024$ pixels, which was con- nected to a graphics board with a refresh rate of $75 \mathrm{~Hz}$. A personal computer (PC) served for controlling stimulus presentation and response registration.

Stimuli. Four different letters, "A", "S", "H," and "E" were used and were mapped to two response categories, ("A," "S") and ("H," "E"). Sixteen compound stimuli were created by combining all letters, where global letters were constructed from identical local letters arranged within a $5 \times 5$ grid. At a viewing distance of $111 \mathrm{~cm}$ the global letters extended a visual angle of $3.3^{\circ}$ horizontally and of $4.5^{\circ}$ vertically, and the local letters extended a visual angle of $0.5^{\circ} \times$ $0.7^{\circ}$, respectively. Two stimulus types were used: line letters and outline letters (Figure 1). Both types were drawn in white on a black background.

Procedure. Subjects were instructed to classify the letters by pressing one out of two response buttons with the index or middle finger, respectively, of the same hand. Gender, mapping of the letter pairs to the response keys (or fingers), and responding hand were counterbalanced across subjects. For instance, some of the subjects had to respond to "A" or "S" with the index finger and to " $\mathrm{H}$ " and "E" with the middle finger of the right hand. For other subjects, the response hand and/or the finger mapping were reversed.

Each trial started with the appearance of a fixation cross and a cue for $306 \mathrm{msec}$. Both were centered on the screen. The cue was the outline of a rectangle, in a size that corresponded to that of the relevant letter level. After a blank screen of $306 \mathrm{msec}$, the stimulus was presented for $93 \mathrm{msec}$ either to the LVF or to the RVF. The stimuli were positioned in such a way that the right or left border aligned with the center of the screen, respectively. We chose this small eccentricity to restrict the degradation of the local letters, and since it is known that there is almost no overlap between the nasal and temporal areas of the human retina (e.g., Lines \& Milner, 1983). The next cue appeared 1,000 $\mathrm{msec}$ after the response. Errors were signaled by a tone.

There were five factors: stimulus type (line, outline), VF (LVF, RVF), consistency (consistent, inconsistent), level (global, local), and level mode (randomized level, constant level). The first three factors were randomized within each block of trials, whereas the last factor was blocked. The target level was blocked or randomized depending on the level mode. Blocks with a constant level alternated with those with randomized levels, where the starting conditions (level, level mode) were balanced across subjects. Altogether there were 32 conditions. After some practice blocks, 12 blocks of 128 trials were run, distributed over two 1-h sessions. Altogether we obtained 48 responses for each condition per subject.

\section{Results}

Response times. The latencies of correct responses were entered into a five-factor analysis of variance (ANOVA) for repeated measurements on all factors: stimulus type (line, outline), VF (LVF, RVF), level (global, local), consistency (consistent, inconsistent), and level mode (constant, randomized).

The analysis revealed a significant main effect of level mode $[F(1,15)=23.7, p<.001]$, indicating that responses in the constant condition were faster $(592 \mathrm{msec})$ than those in the randomized condition $(659 \mathrm{msec})$. Also the consistency factor was significant $[F(1,15)=123, p<$ $.001]$. Consistent stimuli led to faster responses $(611 \mathrm{msec})$ than inconsistent ones $(640 \mathrm{msec})$. Of the two-way interactions, that between stimulus type and level was reliable $[F(1,15)=15.6, p<.01]$. For the outline stimuli, responses to the global level were faster $(22 \mathrm{msec})$ than responses to the local level, whereas no corresponding difference occurred for line stimuli. However, there was a three-way in- 
teraction between stimulus type, level, and consistency $[F(1,15)=22.7, p<.01]$. For the global level, there was more interference from the local level for line stimuli than for outline stimuli (consistency effect for responses to global: line, $41 \mathrm{msec}$; outline, $25 \mathrm{msec}$ ), whereas the opposite held for responses to the local level (consistency effect for responses to local: line, $18 \mathrm{msec}$; outline, $30 \mathrm{msec}$ ).

With respect to hemispheric differences, there was a reliable interaction between stimulus type, visual field, and level $[F(1,15)=6.35, p<.05]$. A further analysis revealed that this interaction was due to a significant $\mathrm{VF}$ effect difference for the local level. For line stimuli there was a local VF effect of $5 \mathrm{msec}$, whereas the corresponding VF effect for outline stimuli was $-5 \mathrm{msec}[t(63)=$ $2.07, p<.05]$. Neither of these effects was significantly different from zero.

The VF-factor was also involved in a four-way interaction between level mode, visual field, level, and consistency $[F(1,15)=4.69, p<.05]$. The data can be seen in Figure 2. In order to decompose this interaction, the data for the two level modes were analyzed separately.

The data for the condition with constant target levels can be seen in the upper panel of Figure 2. The analysis revealed a significant consistency effect $[F(1,15)=73.3$, $p<.001]$. Furthermore, there was a significant interaction between stimulus type and level $[F(1,15)=15.0, p<$ $.01]$ and a three-way interaction between stimulus type, level, and consistency $[F(1,15)=16.8, p<.001]$. The patterns of these effects correspond to those of the main analysis.

The data for the condition with randomized target levels can be seen in the lower panel of Figure 2. As for the condition with constant levels, the consistency factor was significant $[F(1,15)=60.3, p<.001]$. The same holds for the interaction between stimulus type and level $[F(1,15)=9.28, p<.01]$ and for that between stimulus type, level, and consistency $[F(1,15)=9.87, p<.01]$.

Different from the constant-level condition, however, the interaction between VF, level, and consistency was significant $[F(1,15)=6.13, p<.05]$. A further analysis revealed that there was a significant VF effect of $19 \mathrm{msec}$ $[t(31)=2.03, p<.05]$ for inconsistent stimuli, whereas that for consistent stimuli was actually negative $(-3 \mathrm{msec})$ but not significant $[t(31)=-0.303, p=.732]$.

Error rates. Errors occurred, on average, in $3.6 \%$ of the trials. They were subjected to an ANOVA of the same type as the response times. It turned out that the level mode factor was significant $[F(1,15)=23.9, p<.001]$. There were $2.75 \%$ error trials in the blocked condition and $4.44 \%$ in the randomized condition. There was also a reliable consistency effect $[F(1,15)=9.39, p<.01]$. However, consistency interacted significantly with level mode $[F(1,15)=14.9, p<.01]$. For a constant target level, the error rate difference between responses to consistent and inconsistent stimuli was smaller $(2.19 \%$ vs. $3.30 \%)$ than that under randomized levels ( $2.43 \%$ vs. $6.44 \%)$.

Also, the two-way interaction between stimulus type and level was reliable $[F(1,15)=9.52, p<.01]$, indicating that, with respect to outline stimuli, fewer errors were made for global than for local letters (3.30\% vs. $3.95 \%$ ), whereas the opposite held for the line stimuli (3.90\% vs. 3.22\%).

Finally, there was an interaction between VF, level, and consistency $[F(1,15)=7.97, p<.05]$. As for the response times, VF effects in the expected direction occurred only

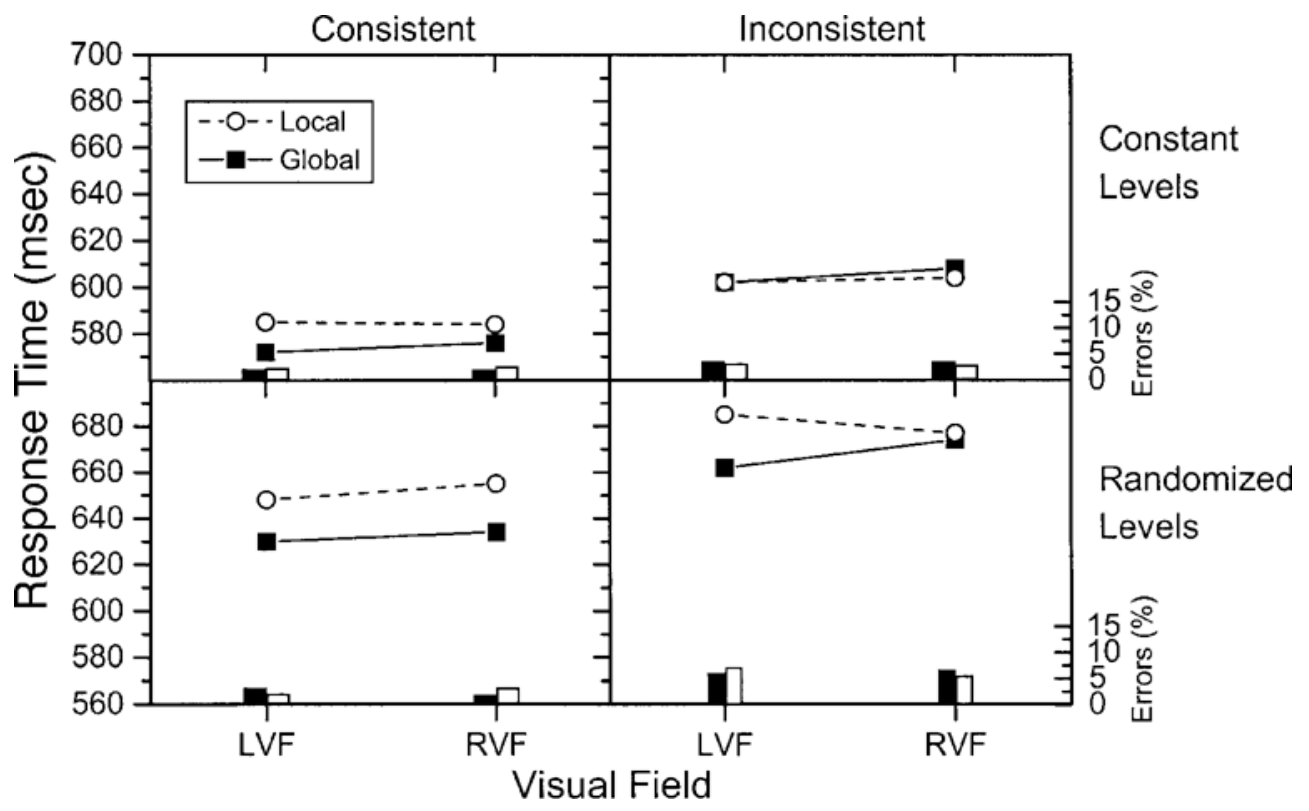

Figure 2. The data showing the four-way interaction between level mode, visual field, level, and consistency in Experiment 1. LVF, left visual field; RVF, right visual field. 
for inconsistentstimuli. However, a further analysis showed that this effect $(1.32 \%)$ was not significant $[t(63)=1.48$, $p=.14]$. On the other hand, the VF effect was significant for consistent stimuli $[t(63)=-2.64, p<.05]$, but it was in the opposite direction $(-1.47 \%)$.

\section{Discussion}

As in Hübner (1997), VF effects in the expected direction occurred only under randomized target levels. However, even for this condition, the effects did not appear for all conditions. Rather, they were restricted to inconsistent stimuli. As hypothesized, the interference was also increased under randomized target levels. Although the consistency effect for the response times increased only slightly, from $25 \mathrm{msec}$ in the blocked condition to $32 \mathrm{msec}$ in the randomized condition, there was a significant increase in the corresponding error rates. Thus, it seems justified to conclude that VF effects for global/local processing are positively related to the absolute amount of interference between the levels.

On the other hand, the induced patterns of relative interference did not affect VF effects in the expected way. Although both stimulus types produced similarly fast responses and about the same amount of absolute interference, they differed, as intended, with respect to their relative amount of interference. That is, for responses to the global level, the line stimuli produced more interference from the local level than did the outline stimuli, whereas for responses to the local level, the outline stimuli produced more interference from the global level than did the line stimuli. If, as the amplifier hypothesis predicts, interference from the nontarget level favor VF effects, then they should have been larger for the global level of the line stimuli than for the global level of the outline stimuli. Analogously, VF effects for responses to the local level should have been larger for the outline stimuli than for the line stimuli. However, there was no significant VF effect difference with respect to the global level between the two stimulus types. Moreover, the obtained significant VF effect difference between the stimulus types for responses to the local level were actually in the opposite direction of that predicted by the amplifier hypothesis.

Taken together, the results of this experiment lend little support to the amplifier hypothesis. Although significant VF effects occurred in the expected direction for inconsistent stimuli, relative interference did not affect VF effects as predicted.

In order to manipulate the relative interference, we used different line types. Our idea was that this should affect the relative salience between the levels, and, consequently, the relative interference. However, by using different stimulus types, we varied not only the relative salience but also other stimulus factors such as the spatial-frequency content, lateral masking, and so on. Thus, we cannot definitively rule out the possibility that other processes affected the VF effects in an uncontrolled and possibly unfavorable way. Therefore, a different method for varying the relative interference was applied in the next experiment.

\section{EXPERIMENT 2}

Is there any method for varying the relative perceptual salience between the levels without simultaneously affecting important low-level stimulus features such as the spatial-frequency content? Recently, Han, He, Yund, and Woods (2001) increased the perceptual salience of the local level by presenting a single element of their hierarchical stimuli in a different color. A similar method was used in the present experiment. We expected that the processing of the local level should benefit from individual local items that pop out and, therefore, attract attention. Consequently, the relative interference between the levels should change for what we call "color" stimuli (Figure 1) in favor of the local level. Thus, with respect to the amplifier hypothesis, the VF effects should vary accordingly. That is, relative to standard stimuli, the VF effects for the local level should decrease for color stimuli, whereas those for the global level should increase.

\section{Method}

Subjects. Sixteen persons (14 female, mean age 24.3 years; 2 male, mean age 36.5 years) participated in the experiment. All were righthanded (by self-report) and had normal or corrected-to-norm al vision.

Apparatus and Stimuli. The apparatus and procedure were the same as in Experiment 1. There were two different types of outline stimuli. One was the same as in the previous experiment, whereas the other type consisted of stimuli whose left- and right-most letters of the middle row were drawn in yellow (Figure 1). Thus, irrespective of whether the stimulus was presented in the RVF or in the LVF, the local letter nearest to fixation was yellow.

There were four factors: VF (RVF, LVF), level (global, local), consistency (consistent, inconsistent), and stimulus type (white, color). The first three factors were randomized within a block of trials, whereas stimulus type was blocked. Altogether there were 16 conditions. After some practice blocks, 6 blocks of 128 trials were run, distributed over a 1-h session. Altogether, we obtained 48 responses for each condition and subject. Half the participants began with 3 blocks of white stimuli, whereas the other half started with 3 blocks of color stimuli.

\section{Results}

Response times. The latencies of correct responses were entered into a four-factor ANOVA for repeated measurements on all factors: stimulus type (white, color), VF (LVF, RVF), level (global, local), and consistency (consistent, inconsistent).

The analysis revealed a significant main effect of consistency $[F(1,15)=55.7, p<.001]$. Responses to consistent stimuli were faster $(666 \mathrm{msec})$ than those to inconsistent ones $(708 \mathrm{msec})$. However, there was an interaction between consistency and level $[F(1,15)=4.78, p<.05]$. Moreover, there was also a three-way interaction between stimulus type, consistency, and level $[F(1,15)=6.71, p<$ $.05]$. Color stimuli produced a relatively large local-toglobal interference, as can be seen in Figure 3.

With respect to VF effects, there was a two-way interaction between VF and level $[F(1,15)=8.00, p<.05]$. However, there was also a three-way interaction between VF, level, and consistency $[F(1,15)=12.8, p<.01]$, as 


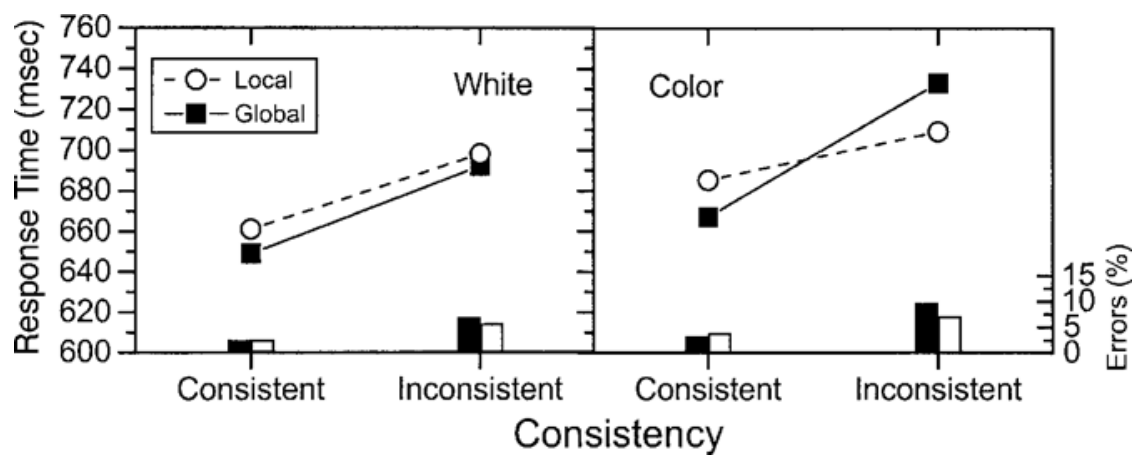

Figure 3. The effect of stimulus type (white vs. color) on the relative consistency effects for the two target levels.

can be seen in Figure 4. A further analysis revealed that a significant VF effect of 44 msec occurred only for inconsistent stimuli $[t(31)=4.28, p<.001]$, whereas the VF effect of $13 \mathrm{msec}$ for consistent stimuli was not significant $[t(31)=0.909, p=.371]$.

Error rates. Errors occurred, on average, in $5.08 \%$ of the trials. They were subjected to an ANOVA of the same type as that for the response times. Of the main factors, only consistency was significant $[F(1,15)=43.4, p<$ $.001]$. Consistent letters produced $2.97 \%$ errors, whereas inconsistent letters produced $7.18 \%$ errors.

However, there was a two-way interaction between level and consistency $[F(1,15)=5.93, p<.05]$, indicating a local interference advantage (consistency effect of $5.30 \%$ for global vs. $3.12 \%$ for local). There was also an interaction between VF and consistency $[F(1,15)=4.87, p<.05]$ (consistency effect for the LVF, 3.44\%; for the RVF, 5.05\%), and between visual field and level $[F(1,15)=5.17, p<$ $.05]$ (VF effect for global, $0.55 \%$; for local, $1.37 \%$ ).

\section{Discussion}

As expected, coloring individuallocal elements increased the local-to-global interference relative to our standard stimuli (Figure 3). However, contrary to the prediction of the amplifier hypothesis, this did not affect the VF effects. To show the independence of VF effects from relative interference, the data for both stimulus types are presented separately in Figure 4. As can be seen, despite the considerable difference of relative interference between the stimulus types, the VF effects were rather similar. On the other hand, the results of the present experiment show again that absolute interference is important for hemispheric asym-

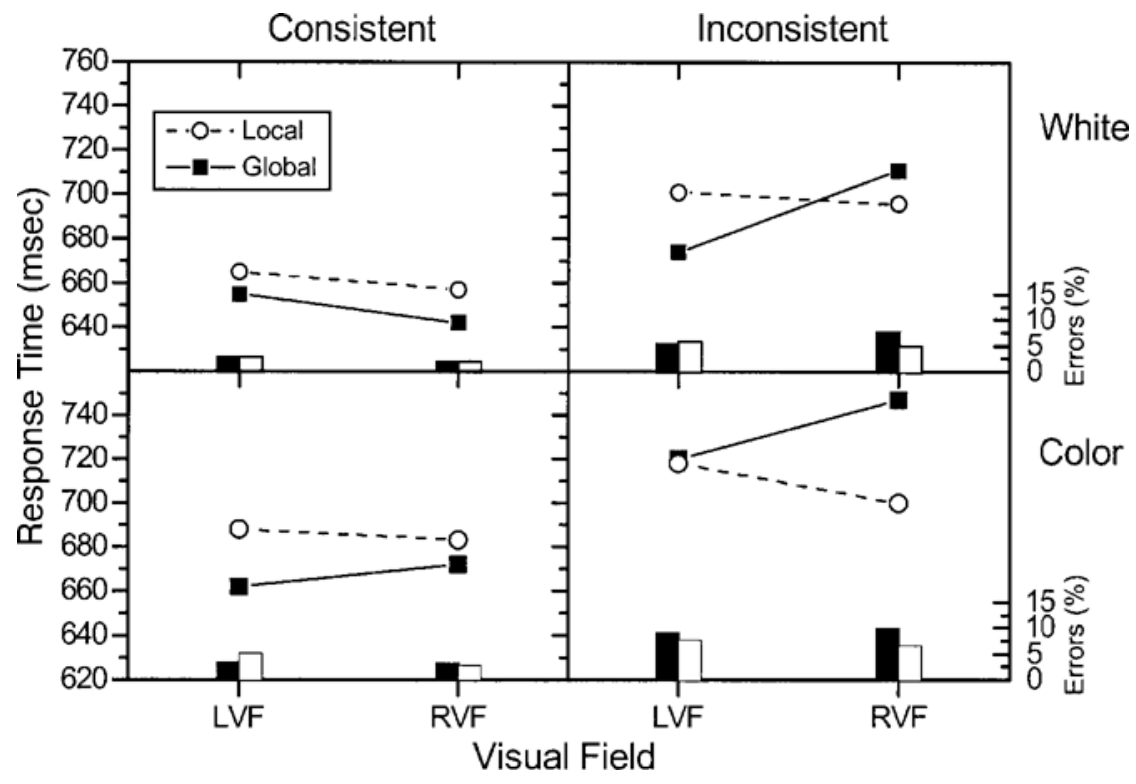

Figure 4. Interaction between stimulus level, visual field, and consistency. As can be seen, this interaction holds for both stimulus types. LVF, left visual field; RVF, right visual field. 
metries with respect to global/local processing. VF effects occurred only for inconsistent stimuli.

Thus, the results of the present experiment also show that there is no quantitative relationship between interference and VF effects. Obviously, a certain minimum amount of absolute interference is sufficient to generate hemispheric differences. This conclusion is at odds with the amplifier hypothesis. However, it is in line with the catalyst hypothesis.

Most global/local experiments employ only two different letters or forms. In this case, consistent stimuli always had the same letter or form at each level. On the other hand, inconsistent stimuli always had different forms at the two levels. Thus, consistent versus inconsistent was confounded with same versus different forms at the stimulus levels. With four letters, as in our case, this confounding was reduced, since two of the four consistent stimuli consisted of different letters. Nevertheless, consistent stimuli still differed from inconsistent ones, because the former comprised two letter combinations with identical letters, which was not the case for the latter type. Therefore, one might ask whether the result that VF effects occurred only for inconsistent stimuli in our experiments was due, at least partly, to the fact that all of these stimuli contained two different letters.

To answer this question, we analyzed the data separately for the two consistent stimulus types, but averaged them across our last two experiments. To distinguish between the different stimulus types, we will use the term congruent for stimuli with identical letters at both levels. On the other hand, response compatible stimuli with different letters will be denoted as compatible. As before, response-incompatible stimuli will be called inconsistent. When VF effects are substantially increased for stimuli with different letters at the levels, they should also be present for compatible stimuli.

The data are shown in Figure 5. As can be seen, there was an interaction between VF, level, and consistency
$[F(2,62)=5.47, p<.01]$. A comparison between the congruent and the compatible stimuli revealed a significant main effect $[F(1,31)=47.4, p<.001]$. Congruent stimuli produced faster responses than did compatible ones (649 vs. $667 \mathrm{msec}$ ). However, there was no interaction with VF. On the other hand, if we compare compatible with inconsistent stimuli, there was not only a significant main effect of consistency $[F(1,31)=44.1, p<.001]$ but also a significant interaction between consistency, level, and $\operatorname{VF}[F(1,31)=5.04, p<.05]$. As further tests revealed, there was a significant $V F$ effect of $32 \mathrm{msec}$ for inconsistent stimuli $[t(63)=4.43, p<.001]$. Although there was a tendency $(9 \mathrm{msec})$ toward VF effects for compatible stimuli, it was far from significant $[t(63)=0.99$, $p=.32]$. Moreover, for the corresponding error rates there was a significant VF effect $[t(63)=-2.28, p<.05]$ in the opposite direction $(-1.50 \%)$.

This analysis clearly shows that the VF effects observed for inconsistent stimuli in our experiments were not simply due to the fact that all of them had a different letter at each level.

\section{EXPERIMENT 3}

The data of our previous experiments do not provide much evidence for the hypothesis that response conflicts simply amplify existing VF effects. Rather, conflicts seem to play a more crucial role, as indicated by our catalyst hypothesis. The catalyst hypothesis is compatible with the lack of any quantitative relationship between the amount of relative interference and the magnitude of the corresponding VF effects.

A further result that seems to be in favor of the catalyst hypothesis is the complete absence of VF effects for consistent stimuli. The amplifier hypothesis is based on the assumption that there are also at least small VF effects for nonconflicting stimuli. Unfortunately, this assumption cannot be directly applied to our consistent stimuli. Quite

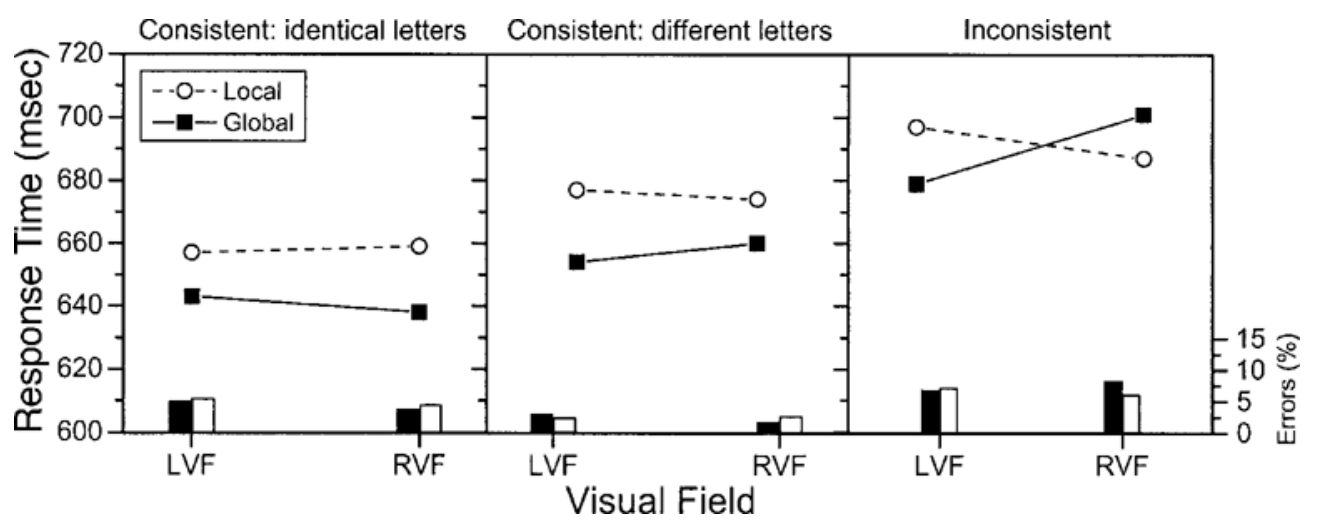

Figure 5. Comparison of the data across Experiments 1 and 2. The data for the consistent condition were analyzed separately for congruent (same letters at both levels) and compatible (different letters at both levels) stimuli. LVF, left visual field; RVF, right visual field. 
the opposite, the amplifier hypothesis even predicts that there will be no VF effects for consistent stimuli. If we assume, as before, that the information at each level contributes to the response, then it follows that the inferior processing of the information at one level can be compensated for by the superior processing of the information at the other level. For instance, if the local level of an LVF stimulus can only be processed suboptimally in the $\mathrm{RH}$, then the optimal processing of the response-compatible global form in the same hemisphere can make up for this deficit. Such a compensation could especially be the case for consistent stimuli that contain the same letter at both levels.

Thus, the same reasoning that predicts an amplification of VF effects for inconsistent stimuli also predicts a reduction of VF effects for consistent stimuli. In order to test whether VF effects also occur without interference, stimuli are needed that prevent any interaction between the levels. A simple way to construct such stimuli is to place a response-irrelevant form at the nontarget level. This was done in Experiment 3. When the amplification hypothesis holds, at least small VF effects should occur for such neutral stimuli.

In order to minimize possible strategy effects, the neutral stimuli were exclusively mixed with consistent stimuli within a block of trials. For comparison, there were also blocks in which inconsistent stimuli were mixed with consistent ones.

\section{Method}

Sixteen persons ( 8 female, mean age 21.5 years; 8 male, mean age 23.9 years) participated in the experiment. All were right-handed (by self-report) and had normal or corrected-to-normal vision. The apparatus and procedure were similar to those in the former experiments. Outline letters served as stimuli. However, additional to consistent stimuli (which in this experiment did not include stimuli with the same letter at both levels) and inconsistent stimuli, neutral stimuli were also presented. The neutral stimuli always had a rectangular form $(\square)$ at the irrelevant level. Three main factors were varied: VF, level, and consistency. However, with respect to consistency there were two different mixed conditions. In one of these blocked conditions neutral stimuli were mixed with consistent ones, whereas in the other condition inconsistent stimuli were mixed with consistent stimuli.

For each mixed condition the three factors were randomized within a block of trials. Thus, altogether there were 16 conditions. After some practice blocks, 12 blocks of 64 trials were run, distributed over a 1-h session. Half the subjects started with 6 neutral blocks and continued with 6 inconsistent blocks. For the other half of the subjects the order was reversed. Altogether we obtained 48 responses for each condition per subject.

\section{Results}

Only latencies of correct responses were entered into two separate three-factor ANOVAs for repeated measurements on all factors.

The first ANOVA analyzed the response times for the condition in which neutral stimuli were mixed with consistent ones. The factors were VF (LVF, RVF), level (global, local), and consistency (consistent, neutral). It turned out that the consistency factor was significant $[F(1,15)=$ $4.66, p<.05]$. However, there was an interaction between level and consistency $[F(1,15)=6.04, p<.05]$, indicating that a consistency effect occurred only for the local level (17 vs. $1 \mathrm{msec})$. No other factor was significant. A similar analysis was performed with the errors. The mean error rate was $5.19 \%$. Only the consistency factor was significant $[F(1,15)=21.2, p<.001](3.63 \%$ for consistent vs. $7.65 \%$ for neutral).

Second ANOVA analyzed the latencies for the condition in which consistent and inconsistent stimuli were mixed. The factors were VF (LVF, RVF), level (global, local), and consistency (consistent, inconsistent). Again, the consistency factor was significant $[F(1,15)=23.9$, $p<.001]$. Responses to consistent stimuli were faster than those to neutral ones (577 vs. $607 \mathrm{msec}$ ). Furthermore, the interaction between $\mathrm{VF}$ and level was significant $[F(1,15)=$ $7.75, p<.05]$. However, as can be seen in Figure 6, the expected data pattern occurred only for inconsistent stimuli. Although the interaction between VF, level, and consistency was only marginally significant $[F(1,15)=3.18$, $p=.095]$, a further analysis with the data subsets was computed. It revealed a significant VF effect of $30 \mathrm{msec}$ for the inconsistent stimuli $[t(15)=3.96, p<.01]$ but no reliable effect $(11 \mathrm{msec})$ for the consistent stimuli $[t(15)=$ $1.13, p=.27]$.

The mean error rate for this mixed condition was $6.86 \%$. The only significant effect was consistency $[F(1,15)=$ $37.3, p<.001]$ ( $2.97 \%$ for consistent vs. $10.7 \%$ for inconsistent).

\section{Discussion}

The results show that the responses to neutral stimuli were faster than those to inconsistent stimuli, but slower than those to consistent ones. This indicates that the responses either benefited or suffered from the information at the nontarget level, depending on its compatibility. However, as before, VF effects were affected only by incompatible information. Neither consistent nor neutral stimuli had any comparable effect. Thus, the fact that there were no VF effects for consistent stimuli in our previous experiments cannot be accounted for by the idea that existing hemispheric asymmetries were counterbalanced by the contribution of the information at the nontarget level. If this hypothesis were valid, at least small VF effects should have occurred for neutral stimuli because their nontarget level contained only response-irrelevant information. Consequently, any efficiency differences between the hemispheres for processing the target level should have been observable.

Given these results, we can reject the amplifier hypothesis. The results again support the catalyst hypothesis, which states that response competition between the levels is crucial for VF effects.

\section{GENERAL DISCUSSION}

The aim of this article was to investigate the question of why VF effects with respect to functional hemispheric differences for global/local processing are rather unreliable. 


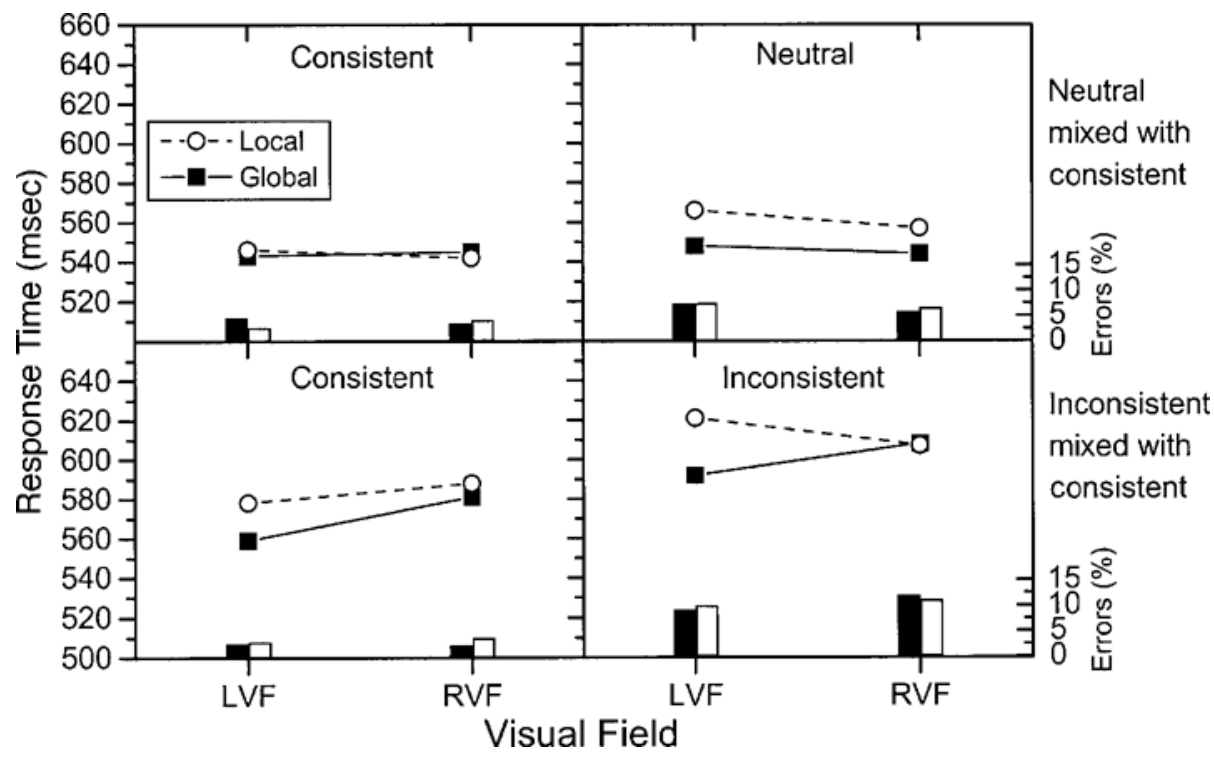

Figure 6. Results of Experiment 3. The conditions in the upper two panels and those in the lower two panels were mixed within a block of trials, respectively.

Although numerous results indicate that the left cerebral hemisphere is more efficient at processing local information whereas the right hemisphere is superior in processing global information, the corresponding VF effects have not shown up consistently in response time studies. Interestingly, in a number of studies these effects occurred mainly for stimuli that induced a response conflict (Hübner, 1997, 1998; Kitterle et al., 1993; Van Kleeck, 1989). Van Kleeck (1989) offered a straightforward explanation for this finding. He assumed that each hemisphere is capable of processing the information at both levels but with different efficiency, and that the results compete for response selection. Since, due to the assumed efficiency differences, the competition for a given target level is considerably stronger for stimuli presented in one VF than for those presented in the other VF, response competition leads to an amplification of existing VF effects.

The present paper reports a series of experiments in which this amplifier hypothesis was tested. As an alternative hypothesis, we also considered the idea that response competition is crucial for VF effects in the sense that it acts as a catalyst rather than as an amplifier.

According to the amplifier hypothesis, there should be a quantitative relation between the amount of response competition or interference between the levels and the size of VF effects. Therefore, in Experiment 1, the extent of interference should have been varied by using constant versus randomized target levels (see Hübner, 1997). As expected, response competition was increased under randomized levels relative to conditions with constant levels. Moreover, reliable VF effects in the expected direction occurred only for inconsistent stimuli under randomized levels, which is in line with the amplifier hypothesis.
A further prediction of the amplifier hypothesis is that not only the absolute amount of competition or interference should affect VF effects but also the relative interference between the levels. That is, when the relative strength of one level is increased at the expense of the other, the corresponding VF effect should decrease for that level, whereas that for the other level should increase. To test this prediction, the relative dominance or perceptual salience (e.g., Fink et al., 1999) of the levels was varied in Experiment 1 by using different line types (line vs. outline) for constructing the stimuli. Although this manipulation did not affect the average amount of interference, it modulated the relative interference. Line stimuli produced more local-to-global interference than did outline stimuli. This difference even affected the VF effect for the local level. However, the effect was in the opposite direction to that predicted by the amplifier hypothesis.

Thus, at least one prediction of the amplifier hypothesis could not be confirmed by the results of Experiment 1 . However, since this failure might have been due to our specific stimuli, the prediction was tested again in Experiment 2, where the perceptual salience of the local level and, consequently, the local-to-globalinterference was increased by presenting individual local letters in a different color. It turned out that this variation did not affect VF effects either. Rather, as in Experiment 1, VF effects occurred only for inconsistent stimuli.

So far, the results of our experiments suggest that a certain minimum amount of absolute response competition has to be present in order for VF effects to appear, whereas the relative interference relations between the levels are irrelevant. At least the second result is at odds with the amplifier hypothesis. Concerning the first result, it is im- 
portant to note that the amplifier hypothesis assumes that the VF effects should also exist, even if small, for nonconflicting stimuli. Obviously, this was not the case for our consistent stimuli. However, these stimuli are special in the sense that they are not only nonconflicting but also produce response facilitation. Since both the global level and the local level are consistent, they activate the same correct response. Consequently, the inferior processing of one level in a given hemisphere could have been compensated for by the superior processing of the other level. Thus, analogous to the reasoning that predicts an amplification of VF effects for inconsistent stimuli, it can be predicted that these effects are reduced for consistent stimuli. In any case, the absence of VF effects for consistent stimuli could not be used to reject the amplifier hypothesis. Rather, this is exactly what one would have expected if this hypothesis holds.

To test whether there are also VF effects for nonconflicting stimuli, we used neutral stimuli in Experiment 3. These stimuli contained only response-irrelevant information at the nontarget level that could not contribute to the response in any systematic way. Consequently, when the absence of VF effects for consistent stimuli in the previous experiments was caused by compensation, VF effects should show up for neutral stimuli. On the other hand, when VF effects depend on response conflicts, they should also be absent for neutral stimuli. The results of Experiment 3 clearly show that there were no VF effects for neutral stimuli. As in the previous experiments, VF effects occurred only for inconsistent stimuli.

Taken together, our results provide little evidence in favor of the amplifier hypothesis. The idea that existing small VF effects are merely amplified by response conflicts is not adequate to explain our pattern of results. Rather, our data indicate that response competition is crucial for observing VF effects. In all of our three experiments VF effects occurred only for inconsistent stimuli. Nevertheless, one might doubt that our results hold in general. It could be that they are valid only for response times obtained with lateralized stimuli. Furthermore, although we have shown that conflicts induce VF effects, it is open whether this really relates to hemispheric asymmetries. To investigate these questions we recently conducted an event-related potential(ERP) study (Malinowski, Hübner, Keil, \& Gruber, 2002) with centrally presented stimuli. It turned out that, in accordance with our response time data, the ERPs were lateralized only for inconsistent stimuli and not for consistent ones.

Thus, we have a relatively broad empirical basis for concluding that competition between the levels of a hierarchical stimulus is favorable for inducing hemispheric asymmetries and for observing corresponding VF effects. Moreover, our results indicate that the relation between response competition and VF effects is qualitative rather than quantitative. This suggests that conflicts operate more like a catalyst than like an amplifier. Unfortunately, although this analogy is helpful for labeling our alternative hypothesis, it does not explain the underlying mechanisms. The question of how interference affects VF ef- fects still remains. It is conceivable that attentional or strategic mechanisms and, eventually, interactions between the hemispheres are involved. For instance, one might assume that resolving response conflicts require some kind of resource management within or between the hemispheres (see Banich \& Weissman, 2000; Weissman \& Banich, 1999; Zaidel \& Rayman, 1994). The neuropsychological account of Robertson and Lamb (1991) points in this direction.

Although these ideas offer a great deal of flexibility for explaining various results, it is hard to see how they could account for our results. Since there is no reason to assume that the involved early perceptual processes are not the same for consistent, neutral, and inconsistent stimuli, we have to assume that response selection constitutes the only difference between these stimulus types. Thus, the crucial question is how VF effects for global/local processing are related to response conflicts. How is it possible that differences between the cerebral hemispheres with respect to a perceptual dimension depend on conflicts that occur at the stage of response selection? Below, we propose a possible explanation.

Consistency effects, as observed in our own as well as in countless other experiments, demonstrate that stimulus features can activate an associated response automatically. This allows one to rely on automatic activation and to respond in a reflexlike manner (see Hommel, 2000). In our experiments, such a responding was possible for consistent stimuli, because the information at both levels activated the same correct response. However, the situation was quite different for inconsistent stimuli. In this case the information was ambiguous and activated competing responses so that response selection had to be based on a different strategy (see Carter et al., 2000). To resolve the response conflict, the subjects inevitably had to take into account the level at which the letters occurred. This could be accomplished only with an elaborated stimulus representation, where letter identity and level were integrated. We hypothesize that the hemispheres differ with respect to this integration process. The identity of the global letter is more efficiently integrated with its level in the $\mathrm{RH}$, whereas the same holds for the identity of the local letter in the LH. Consequently, the hemispheres differ in their efficiency or capacity for the integration of form and level, rather than in the quality of their early stimulus representations. This explains why there were no VF effects for consistent and neutral stimuli.

Thus, the crucial difference between consistent or neutral and inconsistent stimuli is that responses to the former can be based on early and incomplete stimulus representations, whereas those to the latter require later and more complete representations.

The assumption that the hemispheres differ only with respect to their late representations also explains the qualitative relation between conflict and VF effects. When the amount of competition exceeds a certain value, a transition has to occur from the state where early representations are sufficient for responding to a state where complete representations are required. Once the system is in 
this higher stage, a further increase of interference has no additional effects for the hemispheric asymmetries.

Given our account, one might ask whether the introduction of response conflicts is the only way to produce VF effects. This is certainly not the case. Since inconsistent stimuli also produced the slowest responses, one could alternatively hypothesize that VF effects depend on task difficulty. Although we think that an increased task difficulty per se is not sufficient for obtaining VF effects, and that, therefore, this hypothesis is too general, there are certainly other beneficial conditions for VF effects, which probably will also be more difficult than conditions with consistent stimuli. Presumably, various factors such as the learning state, the activation state, the stimulus characteristics, and so on, determine whether the system has to rely on later representations or whether earlier representations are sufficient. As we have seen for the condition with blocked target levels in Experiment 1, when the irrelevant level can be inhibited permanently, an early representation can even be sufficient for responding to inconsistent stimuli.

In sum, the results of the reported experiments support the hypothesis that there are hemispheric asymmetries with respect to global/local processing. However, these asymmetries do not exist for early perceptual stimulus representations. Rather, they seem to exist for later and more elaborated stimulus representations where form and level are integrated. One way to have subjects base their responses on such late representations is to present conflicting stimuli. Whether there are other methods for obtaining this effect must await further research.

\section{REFERENCES}

Banich, M. T., \& Weissman, D. H. (2000). One of 20 questions for the twenty-first century: How do brain regions interact and integrate information? Brain \& Cognition, 42, 29-32.

Boer, L. C., \& Keuss, P. J. G. (1982). Global precedence as a postperceptual effect: An analysis of speed-accuracy tradeoff functions. Perception \& Psychophysics, 31, 358-366.

Boles, D. B. (1984). Global versus local processing: Is there a hemispheric dichotomy? Neuropsychologia, 22, 445-455.

Boles, D. B., \& Karner, T. A. (1996). Hemispheric differences in global versus local processing: Still unclear. Brain \& Cognition, 30, 232-243.

Carter, C. S., Macdonald, A. M., Botvinick, M., Ross, L. L., Stenger, V. A., Noll, D., \& Cohen, J. D. (2000). Parsing executive processes: Strategic vs. evaluative functions of the anterior cingulate cortex. Proceedings of the National Academy of Sciences, 97, 19441948.

Fink, G. R, Halligan, P. W., Marshall, J. C., Frith, C. D., FrackOWIAK, R. S. J., \& Dolan, R. J. (1996). Where in the brain does visual attention select the forest and the trees? Nature, 382, 626-628.

Fink, G. R., Halligan, P. W., Marshall, J. C., Frith, C. D., FrackOWIAK, R. S. J., \& Dolan, R. J. (1997). Neural mechanisms involved in the processing of global and local aspects of hierarchically organized visual stimuli. Brain, 120, 1779-1791.

Fink, G. R., Marshall, J. C., Halligan, P. W., \& Dolan, R. J. (1999). Hemispheric asymmetries in global/local processing are modulated by perceptual salience. Neuropsychologia, 37, 31-40.

Fink, G. R., Marshall, J. C., Halligan, P. W., Frith, C. D., \& FrackOWIAK, R. (1997). Hemispheric specialization for global and local processing: The effect of stimulus category. Proceedings of the Royal Society of London: Series B, 264, 487-494.

Han, S., He, X., Yund, E. W., \& Woods, D. L. (2001). Attentional se- lection in the processing of hierarchical patterns: An ERP study. Biological Psychology, 56, 113-130.

Heinze, H. J., Hinrichs, H., Scholz, M., Burchert, W., \& Mangun, G. R. (1998). Neural mechanisms of global and local processing: A combined PET and ERP study. Journal of Cognitive Neuroscience, 10, 485-498.

Hommel, B. (2000). The prepared reflex: Automaticity and control in stimulus-response translation. In S. Monsell \& J. Driver (Eds.), Control of cognitive processes: Attention and performance XVIII (pp. 247273). Cambridge, MA: MIT Press.

HÜBNER, R. (1997). The effect of spatial frequency on global precedence and hemispheric differences. Perception \& Psychophysics, 59, 187201.

HÜBNER, R. (1998). Hemispheric differences in global/local processing revealed by same-different judgements. Visual Cognition, 5, 457-478.

Humphreys, G. W., \& Boucart, M. (1997). Selection by color and form in vision. Journal of Experimental Psychology: Human Perception \& Performance, 23, 136-153.

IVRY, R. B., \& RoberTSON, L. C. (1998). The two sides of perception. Cambridge, MA: MIT Press.

Kitterle, F. L., Christman, S., \& Conesa, J. (1993). Hemispheric differences in the interference among components of compound gratings. Perception \& Psychophysics, 54, 785-793.

Lamb, M. R., \& Robertson, L. C. (1988). The processing of hierarchical stimuli: Effects of retinal locus, locational uncertainty, and stimulus identity. Perception \& Psychophysics, 44, 172-181.

Lines, C. R., \& Milner, A. D. (1983). Nasotemporal overlap in the human retina investigated by means of simple reaction time to lateralized light flash. Experimental Brain Research, 50, 166-172.

Livingstone, M., \& Hubel, D. (1988). Segregation of form, color, movement, and depth: Anatomy, physiology, and perception. Science, 240, 740-749.

Malinowski, P., Hübner, R., Keil, A., \& Gruber, T. (2002). The influence of response competition on cerebral asymmetries for processing hierarchical stimuli revealed by ERP recordings. Experimental Brain Research, 144, 136-139.

Martinez, A., Moses, P., Frank, L., Buxton, R., Wong, E., \& Stiles, J. (1997). Hemispheric asymmetries in global and local processing: Evidence from fMRI. NeuroReport, 8, 1685-1689.

Miller, J. (1981). Global precedence in attention and decision. Journal of Experimental Psychology: Human Perception \& Performance, 7, 1161-1174.

NAVON, D. (1977). Forest before the trees: The precedence of global features in visual perception. Cognitive Psychology, 9, 353-393.

Robertson, L. C., Egly, R., Lamb, M. R., \& Kerth, L. (1993). Spatial attention and cuing to global and local levels of hierarchical structure. Journal of Experimental Psychology: Human Perception \& Performance, 19, 471-487.

Robertson, L. C., \& LAMB, M. R. (1991). Neuropsychological contributions to theories of part/whole organization. Cognitive Psychology, 23, 299-330.

Treisman, A. (1988). Features and objects: The fourteenth Bartlett memorial lecture. Quarterly Journal of Experimental Psychology [A], 40, 201-237.

VAN KLEECK, M. H. (1989). Hemispheric differences in global versus local processing of hierarchical visual stimuli by normal subjects: New data and a meta-analysis of previous studies. Neuropsychologia, 27, 1165-1178.

Weissman, D. H., \& BANICH, M. T. (1999). Global-local interference modulated by communication between the hemispheres. Journal of Experimental Psychology: General, 128, 283-308.

ZAIDEL, E., \& RAYMAN, J. (1994). Interhemispheric control in the normal brain: Evidence from redundant bilateral presentations. In C. Umiltà \& M. Moscovitch (Eds.), Attention and performance XV: Conscious and nonconscious information processing (pp. 477-504). Cambridge, MA: MIT Press.

ZeKI, S., \& SHIPP, S. (1988). The functional logic of cortical connections. Nature, 335, 311-317.

(Manuscript received January 23, 2001; revision accepted for publication January 14, 2002.) 